\title{
Glycyrrhetinic acid-functionalized mesoporous silica nanoparticles as hepatocellular carcinoma- targeted drug carrier
}

This article was published in the following Dove Press journal:

International Journal of Nanomedicine

12 June 2017

Number of times this article has been viewed

\author{
Yongjiu Lv* \\ Jingjing $\mathrm{Li}^{*}$ \\ Huali Chen \\ Yan Bai \\ Liangke Zhang \\ Chongqing Key Laboratory of \\ Biochemistry and Molecular \\ Pharmacology, Chongqing Research \\ Center for Pharmaceutical \\ Engineering, College of Pharmacy, \\ Chongqing Medical University, \\ Chongqing, China \\ *These authors contributed equally \\ to this work
}

\begin{abstract}
In this study, a glycyrrhetinic acid-functionalized mesoporous silica nanoparticle (MSN-GA) was prepared for active tumor targeting. MSN-GA exhibited satisfactory loading capacity for insoluble drugs, uniform size distribution, and specific tumor cell targeting. Glycyrrhetinic acid, a hepatocellular carcinoma-targeting group, was covalently decorated on the surface of MSN via an amido bond. The successful synthesis of MSN-GA was validated by the results of Fourier transform infrared spectroscopy, transmission electron microscopy (TEM), and zeta potential measurement. TEM images revealed the spherical morphology and uniform size distribution of the naked MSN and MSN-GA. Curcumin (CUR), an insoluble model drug, was loaded into MSN-GA (denoted as MSN-GA-CUR) with a high-loading capacity ( $8.78 \% \pm 1.24 \%$ ). The results of the in vitro cellular experiment demonstrated that MSN-GA-CUR significantly enhanced cytotoxicity and cellular uptake toward hepatocellular carcinoma (HepG2) cells via a specific GA receptor-mediated endocytosis mechanism. The results of this study provide a promising nanoplatform for the targeting of hepatocellular carcinoma.
\end{abstract}

Keywords: active tumor targeting, silica nanoparticle, drug loading capacity, HepG2 cells

\section{Introduction}

Hepatocellular carcinoma (HCC) is one of the most common malignancies in the world. ${ }^{1}$ Surgery, chemotherapy, and radiotherapy are the major conventional therapies for tumor, ${ }^{2}$ but they are not suitable for HCC treatment because HCC is typically chemo- and radio-resistant. Moreover, these therapeutic approaches also have numerous limitations and drawbacks, such as strong systemic toxicity and complications, poor compliance, drug resistance, low-postoperative survival time, and high-recurrence rates. ${ }^{3,4}$ Therefore, more novel therapeutic strategies are urgently needed.

Nanoparticle drug delivery systems (NDDS) have been extensively researched over the past few decades and have become the main focus in the development of cancer-targeted theranostics. ${ }^{5,6}$ NDDS alters the biodistribution and pharmacokinetics properties of drugs to ameliorate side effects while enhancing therapeutic effects. These positive effects occur via various mechanisms, such as specific binding to tumor or blood vessel cells, enhanced permeability and retention (EPR) effect, and utilizing the unique pathophysiology and microenvironment of tumors (such as pH-, redox-, enzyme-, or other stimuli-sensitive nanoparticles). ${ }^{7-9}$

Among different nanoparticles, mesoporous silica nanoparticle (MSN) has attracted considerable attention due to its easily modifiable surface, unique morphological characteristics, extensive surface area, high pore volume, and good stability. ${ }^{10-14}$ 
Glycyrrhetinic acid (GA) and glycyrrhizin (GL) are present in the roots of Glycyrrhiza glabra L. Recent studies have found that GA and GL possess pharmacological actions, such as anti-inflammatory, anti-diabetic, and anti-tumor effects. ${ }^{15}$ Furthermore, the surface of rat hepatocyte membranes has specific binding sites for GA and GL, ${ }^{16}$ where the number of GA-binding sites is greater than that of GL. Moreover, tumor tissues possess 1.5- to 5-fold more GA receptors than normal tissue. ${ }^{17,18}$ Curcumin (CUR) is a diketone compound that is derived from Curcuma longa. Modern pharmacological studies have revealed the numerous pharmacological activities of CUR, such as anti-inflammation, antioxidation, anti-cancer, and antiangiogenesis activities. ${ }^{19,20}$ Moreover, CUR inhibits the proliferation of HCC cells and has antiangiogenic activity against HCC cells that were implanted in nude mice. ${ }^{21,22}$ However, the poor water solubility and bioavailability of CUR limit its clinical applications. ${ }^{23}$ These limitations can be addressed by hosting CUR in the mesopores of highly drug-loaded MSN.

In this study, GA was selected as a target ligand and was covalently modified on the surface of MSN (Figure 1). The resultant glycyrrhetinic acid-functionalized mesoporous silica nanoparticle (MSN-GA) has the potential to specifically deliver drugs to HCC cells. CUR, as an insoluble anticancer drug, was

A
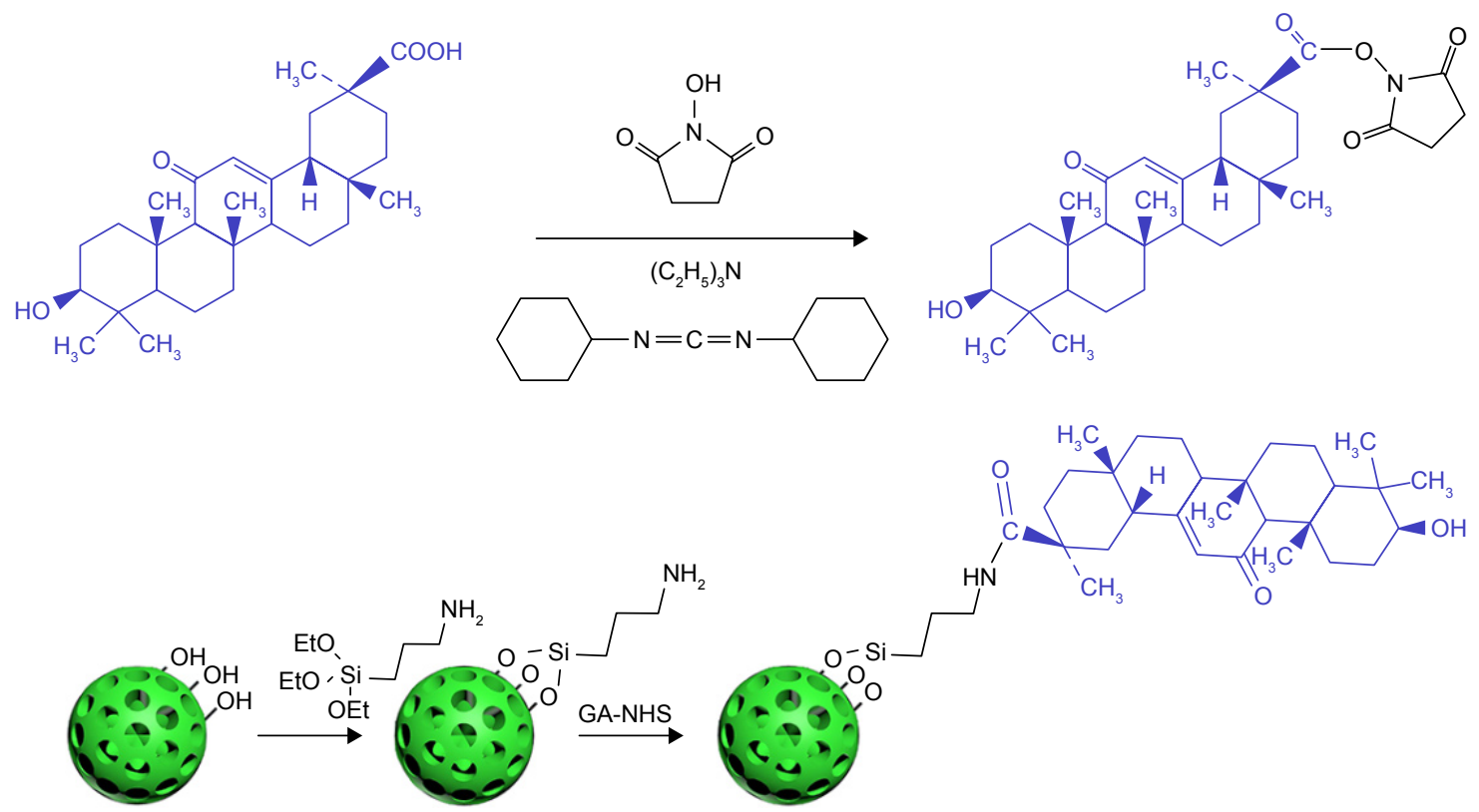

B

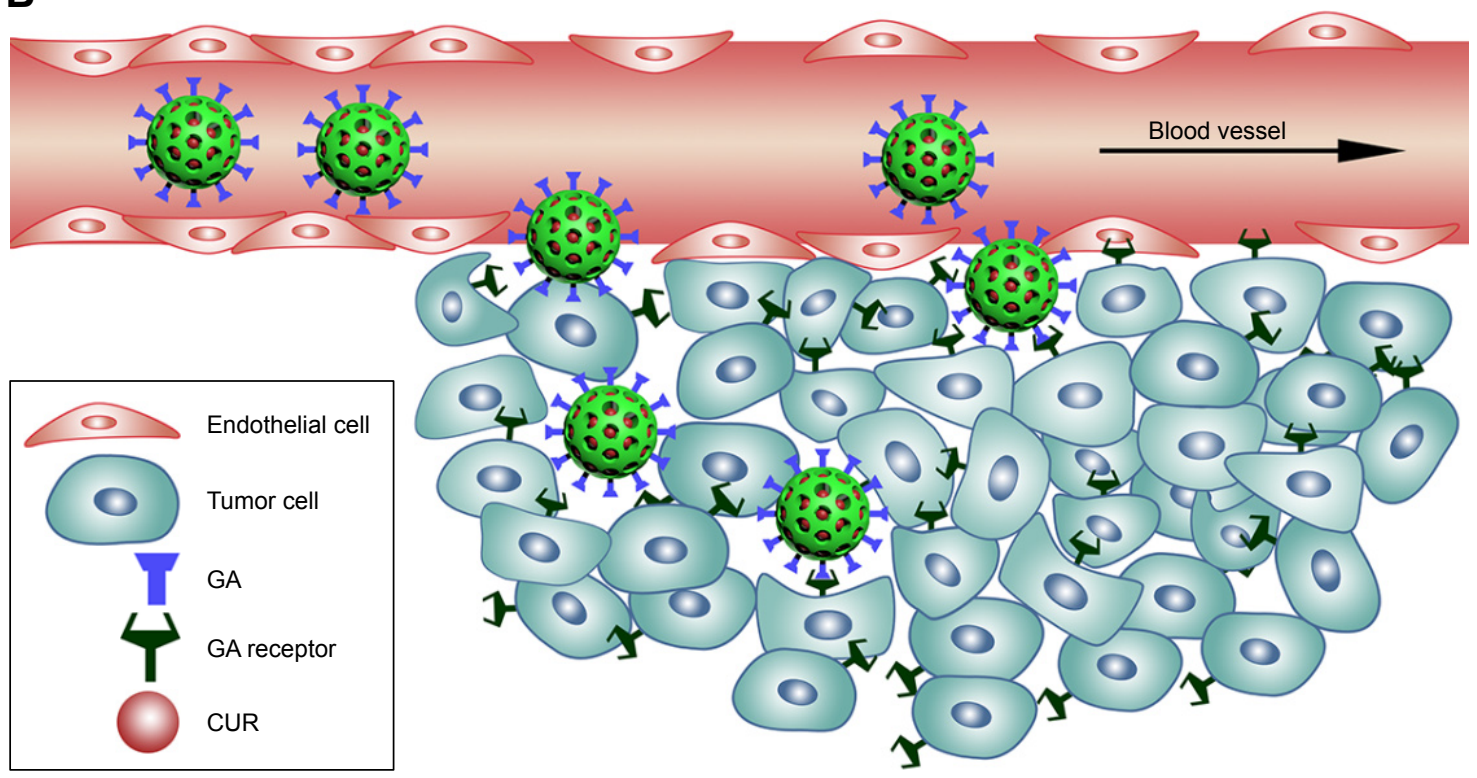

Figure I Synthesis of MSN-GA (A) and schematic of MSN-GA for HCC-cell targeting (B).

Abbreviations: GA, glycyrrhetinic acid; HCC, hepatocellular carcinoma; MSN, mesoporous silica nanoparticle; NHS, N-hydroxysuccinimide. 
loaded into MSN-GA. The covalent bond between GA and MSN was investigated by Fourier transform infrared spectroscopy (FTIR). The characteristics and morphologies of MSN and MSN-GA were studied with transmission electron microscopy (TEM) and dynamic light scattering (DLS). HepG2 cells were selected for the investigation of the cytotoxicity and tumor cell targeting efficiency of CUR-loaded MSN-GA.

\section{Materials and methods Materials}

The following materials were obtained from commercial suppliers and used as received: CUR, GA (98\%), coumarin-6, 4',6-diamidino-2-phenylindole (DAPI), anhydrous dimethyl sulfoxide (DMSO), $N$-hydroxysuccinimide (NHS), $N, N^{\prime}$ dicyclohexyl carbodiimide (DCC), cetyltrimethyl ammonium bromide (CTAB), (3-aminopropyl)-triethoxysilane (APTES), and tetraethyl orthosilicate (TEOS) were all obtained from Aladdin Industrial Corporation (Shanghai, China). DMEM and Trypsin-EDTA solution $(0.25 \%)$ were both supplied by Thermo Fisher Scientific Biological Chemical Co., Ltd. (Beijing, China). Tetrazolium salt (MTT) was purchased from Biosharp Biotechnology Co., Ltd. (Beijing, China). Penicillin and streptomycin (PS) solutions were both obtained from Beijing Dingguo Changsheng Biotechnology Co., Ltd. (Beijing, China). Fetal bovine serum (FBS) was supplied by Zhejiang Tianhang Biotechnology Co., Ltd. (Zhejiang, China). Unless otherwise stated, all solvents were of analytical grade and obtained from Chengdu Kelong Chemical Co., Ltd. (Chengdu, China).

\section{Preparation of MSN}

MSN was synthesized in accordance with reported works. ${ }^{14,24}$ Briefly, CTAB (1.00 g) was added to $480 \mathrm{~mL}$ of deionized water. The suspension was stirred at $80.0^{\circ} \mathrm{C}$ until homogenous. A total of $3.5 \mathrm{~mL} \mathrm{NaOH}(2.00 \mathrm{M})$ was added to the solution under stirring for $10 \mathrm{~min}$, then $5 \mathrm{~mL}$ TEOS was added dropwise. The resultant reaction mixture was stirred for $3 \mathrm{~h}$ to yield a white precipitate. The mixture was continuously stirred at room temperature for $24 \mathrm{~h}$. After centrifugation $\left(10,000 \mathrm{rpm}, 10 \mathrm{~min}, 25^{\circ} \mathrm{C}\right)$, the precipitate was washed six times with deionized water and ethanol, and then dried in a vacuum oven at $60.0^{\circ} \mathrm{C}$.

After synthesis, $0.4 \mathrm{~g}$ of the as-prepared particles were briefly treated with $32 \mathrm{~mL}$ methanol under intensive stirring for $36 \mathrm{~h}$ at $60.0^{\circ} \mathrm{C}$ to remove the template $\mathrm{CTAB}$ from the MSN. Then, $0.2 \mathrm{~mL}$ concentrated hydrochloric acid (36.5\%) was added dropwise to the above suspension. Finally, the suspension was washed six times with methanol. MSN without CTAB was vacuum-dried at $50.0^{\circ} \mathrm{C}$ for $24 \mathrm{~h}$.

\section{Synthesis of amino-functionalized MSNs $\left(\mathrm{MSN}-\mathrm{NH}_{2}\right.$ )}

Amino-functionalized MSN (MSN-NH$)$ was synthesized according to a published method: $0.40 \mathrm{~g} \mathrm{MSN}$ and $1.50 \mathrm{~mL}$ APTES were added to $25 \mathrm{~mL}$ ethanol. ${ }^{25}$ The mixture was stirred for $24 \mathrm{~h}$ at $30^{\circ} \mathrm{C}$. The synthesized $\mathrm{MSN}-\mathrm{NH}_{2}$ was collected by centrifugation, washed six times with ethanol, and vacuum dried at $60.0^{\circ} \mathrm{C}$ for $24 \mathrm{~h}$.

\section{Synthesis of MSN-GA}

MSN-GA was synthesized as follows: $0.52 \mathrm{~g} \mathrm{NHS,} 0.94 \mathrm{~g}$ DCC, $1.00 \mathrm{~g} \mathrm{GA}$, and $0.50 \mathrm{~mL}$ triethylamine were added to $20 \mathrm{~mL}$ anhydrous DMSO. The reaction mixture was stirred for $24 \mathrm{~h}$ at $30^{\circ} \mathrm{C}$ in the dark. The product was filtered and washed with diethyl ether for six times to obtain the NHS ester of GA (GA-NHS) (Figure 1).

Synthesized GA-NHS (60.0 mg) and MSN-NH (100.0 mg) were added to $15 \mathrm{~mL}$ anhydrous DMSO. The mixture was stirred for $24 \mathrm{~h}$ at $60^{\circ} \mathrm{C}$. The synthesized MSN-GA was collected by centrifugation. The unconjugated GA-NHS molecules were removed by washing MSN-GA six times with DMSO and ethanol.

\section{Loading capacity of CUR in MSN}

To investigate the loading capacity of CUR on MSN, $20 \mathrm{~mL}$ ethanol solution of CUR ( $2 \mathrm{mg} / \mathrm{mL})$ was placed in a $50-\mathrm{mL}$ round-bottomed flask with $40 \mathrm{mg}$ MSN. The mixture was dispersed via sonication. Then, ethanol was volatilized with a rotary evaporator at $150 \mathrm{rpm}$ and $50^{\circ} \mathrm{C}$. The final powder was washed with $50 \%$ ethanol $(40 \mathrm{~mL})$ to remove surplus CUR. The dried CUR-loaded MSN was thus acquired (MSN-CUR). The preparation of CUR-loaded MSN-GA (denoted as MSN-GA-CUR) followed the same method as MSN-CUR. Loading capacity was quantified by measuring the absorbance values of the loaded drug in the preparation with a UV-spectrophotometer (UV-5200PC; Shanghai Metash Instruments Co., Ltd., Shanghai, China) at $430 \mathrm{~nm}$ (Eq. (1)).

$$
\mathrm{LC}=\frac{\text { Weight of CUR in preparation }}{\text { Weight of preparation }} \times 100 \%
$$

\section{In vitro drug release}

The in vitro release of CUR from free CUR, MSN-CUR, or MSN-GA-CUR was performed using the dialysis method. Four milliliters of different CUR-loaded preparations that contained $0.3 \mathrm{mg}$ CUR was placed in a dialysis bag (MWCO 
$8,000$ to 14,000$)$. The dialysis bag was immersed in $100 \mathrm{~mL}$ $30 \%$ ethanol solution and incubated in a thermostatic water bath oscillator (Shanghai Samsung Laboratory Instrument Co., Ltd., Shanghai, China) at $37^{\circ} \mathrm{C}$ and $100 \mathrm{rpm}$. At set intervals, a 4-mL sample was taken and replaced with fresh dissolution medium. The sample was filtered through a $0.22-\mu \mathrm{m}$ filter and quantified via UV-Vis spectrophotometry at $430 \mathrm{~nm}$.

\section{Characterization of preparations}

The FTIR spectra of MSN and MSN-GA were obtained using an FTIR spectrophotometer (Nicolet iS50; Thermo Fisher Scientific, Waltham, MA, USA) to confirm the successful synthesis of $\mathrm{MSN}_{-\mathrm{NH}_{2}}$ and MSN-GA. Malvern Zetasizer Nano ZS ZEN 3600 instrument (Malvern, UK) was employed to measure the size distribution and zeta potential of different samples. TEM (Hitachi-7500, Hitachi, Tokyo, Japan) was used to observe the morphology of MSN and MSN-GA. Differential scanning calorimetry (DSC) curves were obtained using a STA449 thermal analyzer (Netzsch, Germany) in the temperature range of $20^{\circ} \mathrm{C}-300^{\circ} \mathrm{C}$ at a heating rate of $10^{\circ} \mathrm{C} \mathrm{min}^{-1}$.

\section{Cell culture and determination of nanoparticle cytotoxicity}

The hepatocellular carcinoma HepG2 cell line was provided by Chongqing Key Laboratory of Biochemistry and Molecular Pharmacology. The cells were cultured in RPMI-1640 medium that contained $10 \% \mathrm{FBS}$ and $1 \%$ PS in a humidified incubator with $5 \% \mathrm{CO}_{2}$ atmosphere. This research has been reviewed and approved by the institutional review board of the Chongqing Medical University.

The cytotoxicity of the nanoparticles against HepG2 cells was determined by MTT assay. HepG 2 cells $\left(5 \times 10^{3}\right.$ cells/well $)$ were seeded into a 96-well plate and cultured for $24 \mathrm{~h}$. Then, the cells were treated with $100 \mu \mathrm{L}$ of fresh medium that contained CUR concentrations of $6,12,24,36,48$, or $60 \mu \mathrm{g} / \mathrm{mL}$. After $24 \mathrm{~h}$ of incubation, $20 \mu \mathrm{L}$ MTT solution $(5 \mathrm{mg} / \mathrm{mL})$ was added per well. The cells were incubated for $4 \mathrm{~h}$. Then, $150 \mu \mathrm{L}$ DMSO was added to each well to dissolve formazan crystals. The absorbance per well was measured with an ELx800 microplate reader (Biotek Inc., Winooski, VT, USA) at $490 \mathrm{~nm}$.

\section{In vitro cellular uptake}

High-performance liquid chromatography (HPLC) and confocal laser scanning microscopy (CLSM) were adopted to evaluate the in vitro uptake of various formulations by HepG2 cells. For HPLC, HepG2 cells $\left(5 \times 10^{3}\right.$ cells/well $)$ were seeded into a six-well plate for $24 \mathrm{~h}$. The cells were treated with free CUR, MSN-CUR, and MSN-GA-CUR for $4 \mathrm{~h}$ and then washed three times with fresh PBS solution to remove non-associated nanoparticles. Then, the cells were collected, suspended in $200 \mu \mathrm{L}$ water, and frozen thrice. After centrifugation $(12,000 \times g, 20 \mathrm{~min}), 1.5 \mathrm{~mL}$ ethyl acetate was added to the collected supernatant and vortexed. The mixture was centrifuged, and the precipitate was then collected and vacuum-dried. Aliquots of the samples were analyzed with HPLC, which was equipped with a Hypersil ${ }^{\text {TM }}$ BDS C18 column $(15 \mathrm{~cm} \times 4.5 \mathrm{~mm}, 5 \mu \mathrm{m})$ and an ultravioletvisible detector set at $430 \mathrm{~nm}$. The mobile phase comprised methanol/water/citric acid (73/26.7/0.3), and the flow rate was $1.0 \mathrm{~mL} / \mathrm{min}$.

For CLSM, HepG 2 cells $\left(6 \times 10^{4}\right.$ cells/well) were seeded for $24 \mathrm{~h}$ in a 24 -well plate that contained one glass coverslip per well. After $30 \mathrm{~min}$ of co-incubation with $100 \mu \mathrm{g} / \mathrm{mL}$ of coumarin-6-labeled MSN (C-6-MSN) or coumarin-6-labeled MSN-GA (C-6-MSN-GA) $37^{\circ} \mathrm{C}$, the cells were washed with PBS, fixed with $3.7 \%$ formaldehyde and stained with DAPI. The glass coverslip was observed under a confocal microscope (Nikon A1R, Japan).

C-6-MSN and C-6-MSN-GA were prepared as follows: $25 \mathrm{mg}$ MSN or MSN-GA were dispersed in $4 \mathrm{~mL}$ coumarin-6 (15 $\mu \mathrm{g} / \mathrm{mL}$ ) chloroform solution. The chloroform was then evaporated using a rotary evaporator to obtain dried C-6MSN or C-6-MSN-GA.

\section{Apoptosis measurement}

Flow cytometry (FCM) was used to analyze apoptosis using an FCM instrument (BD FACS Vantage SE). For apoptosis analysis, HepG2 cells $\left(5 \times 10^{4}\right.$ cells/well) were seeded into a six-well plate. After $24 \mathrm{~h}$ of treatment with free CUR, MSN-CUR, MSN-GA-CUR, or MSN-GA-CUR with free GA, the treated cells were collected and washed with PBS, resuspended in PBS, and stained with Annexin V-fluorescein isothiocyanate (FITC-V) and propidium iodide (PI). Then, the cells were analyzed by FCM.

\section{Statistical analysis}

Data were expressed as mean \pm SD and analyzed by SPSS 13.0 software (IBM Corp., Armonk, NY, USA). $P<0.05$ was considered statistically significant.

\section{Results and discussion Synthesis and characterization of nanoparticles}

The mesoporous silica coating was formed via the selfassembly of the surfactant template-CTAB and silica 
precursor TEOS in basic solution. To generate mesopores, the template-CTAB was removed by a commonly used extraction procedure that used methanol and hydrochloric acid as solvent.

MSN-NH $\mathrm{N}_{2}$ was synthesized in anhydrous organic solvent by the condensation reaction between the silanol group in MSN and oxyethyl group in APTES.

To confirm that the template-CTAB in MSN was completely removed and that MSN-NH${ }_{2}$ and MSN-GA were successfully synthesized, the FTIR spectra of the samples were obtained (Figure 2). The peaks at 2,922 $\mathrm{cm}^{-1}$ and $2,855 \mathrm{~cm}^{-1}$ in the spectra of MSN with CTAB were considered as the $\mathrm{C}-\mathrm{H}$ stretching vibration of CTAB. These peaks disappeared in the spectra of MSN, which indicated the complete removal of the template-CTAB from MSN. All samples showed the framework absorption bands of siliceous materials, such as $\mathrm{Si}-\mathrm{O}-\mathrm{Si}$ asymmetric stretching vibration $\left(1,085 \mathrm{~cm}^{-1}\right)$, $\mathrm{Si}-\mathrm{O}-\mathrm{Si}$ symmetric stretching vibration $\left(800 \mathrm{~cm}^{-1}\right)$, and $\mathrm{Si}-\mathrm{OH}$ stretching vibration $\left(960 \mathrm{~cm}^{-1}\right)$. For MSN-NH ${ }_{2}$, new peaks that were positioned at $2,922 \mathrm{~cm}^{-1}$ and $2,855 \mathrm{~cm}^{-1}$ were considered as the $\mathrm{C}-\mathrm{H}$ stretching vibration of APTES grafted on MSN-NH$H_{2}$. These peaks implied the successful grafting of APTES on MSN (MSN-NH ${ }_{2}$ ).

In the MSN-GA spectrum, the new bands that appeared at $1,447 \mathrm{~cm}^{-1}$ and $1,700 \mathrm{~cm}^{-1}$ were assigned to the amide II band and $\mathrm{C}=\mathrm{O}$ stretching, respectively. Meanwhile a new peak at $1,606 \mathrm{~cm}^{-1}$ was considered as the $\mathrm{C}=\mathrm{C}$ stretching vibration of GA. These new peaks confirmed that the

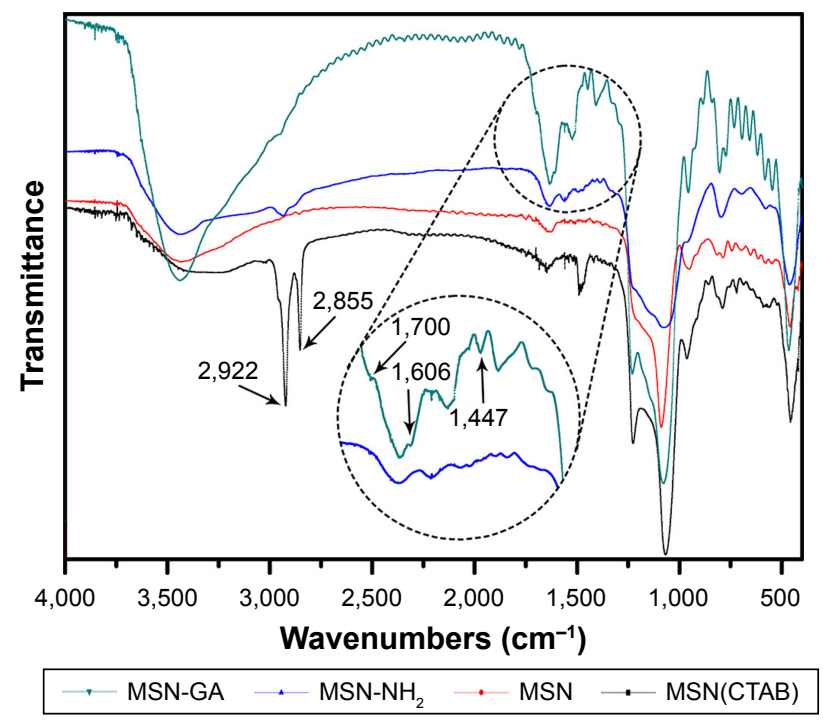

Figure 2 FTIR spectra of MSN(CTAB), MSN, MSN-NH${ }_{2}$, and MSN-GA. Abbreviations: $C T A B$, cetyltrimethyl ammonium bromide; FTIR, Fourier transform infrared spectroscopy; GA, glycyrrhetinic acid; MSN, mesoporous silica nanoparticle; MSN(CTAB), MSN with the template-CTAB.
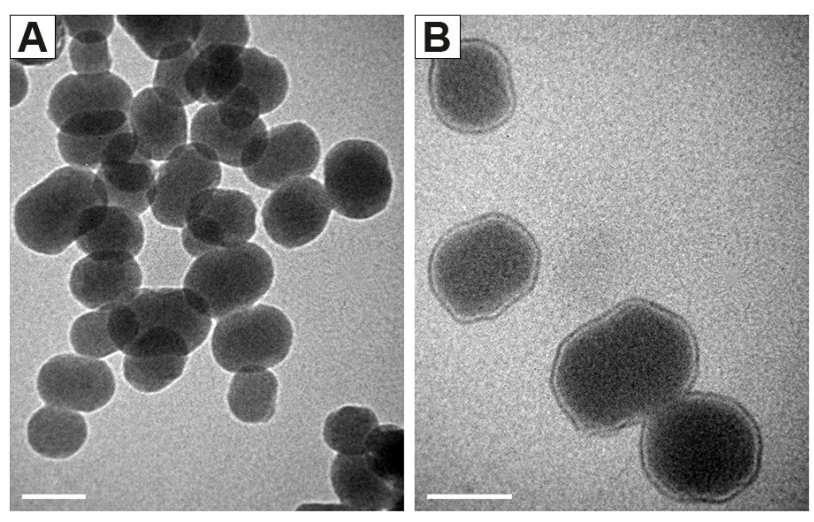

Figure 3 Transmission electron microscopy images of MSN (A) and MSN-GA (B). Scale bar: $100 \mathrm{~nm}$. Magnification $\times 200,000$.

Abbreviations: GA, glycyrrhetinic acid; MSN, mesoporous silica nanoparticle.

GA molecule was covalently grafted onto MSN through an amide bond. ${ }^{26,27}$

The morphology and particle size of the as-synthesized MSN and MSN-GA were characterized with TEM. The TEM images (Figure 3) showed the uniform morphology of MSN and MSN-GA with an average particle size of $\sim 110 \mathrm{~nm}$.

The zeta potential of MSN, MSN-NH ${ }_{2}$, and MSN-GA were measured by Malvern Zetasizer. As shown in Figure 4, the potentials of naked MSN and $\mathrm{MSN}^{-\mathrm{NH}_{2}}$ were $-24 \pm 4.61 \mathrm{mV}$ and $-4.08 \pm 2.56$, respectively. Moreover, the potential of MSN-GA changed to $-25.27 \pm 0.57$ when GA molecule was modified on the surface of MSN. These changes in zeta potential indirectly confirmed the successful synthesis of $\mathrm{MSN}_{-} \mathrm{NH}_{2}$ and MSN-GA.

MSN-GA showed a high drug-loading capacity because of its remarkably high pore volume $\left(0.9 \mathrm{~cm}^{3} / \mathrm{g}\right)$ and the special characteristics of MSN. ${ }^{28}$ The LC of MSN was

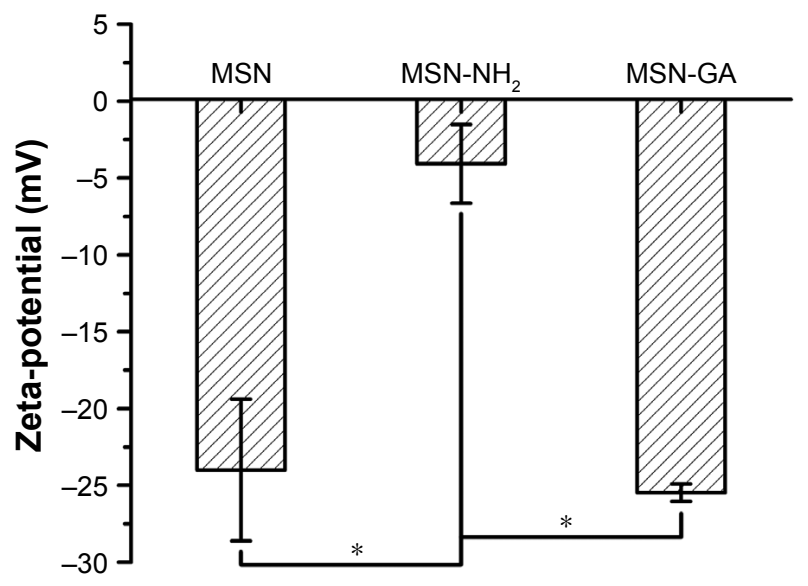

Figure 4 Zeta potentials of MSN, MSN-NH$H_{2}$, and MSN-GA. Note: $* P<0.01$.

Abbreviations: GA, glycyrrhetinic acid; MSN, mesoporous silica nanoparticle. 
$8.78 \% \pm 1.24 \%$. After modifying the surface of MSN with GA, the LC of the resultant MSN-GA was $8.52 \% \pm 0.61 \%$.

DSC was performed to confirm the crystalline/amorphous state of CUR in MSN or MSN-GA. The DSC curves of CUR, physical mixture of MSN and CUR (MSN+CUR), MSN-CUR, and MSN-GA-CUR are shown in Figure 5. The thermograms of CUR and the physical mixture of MSN and CUR (MSN+CUR) displayed a characteristic single-melting endothermic peak at $180^{\circ} \mathrm{C}$, which disappeared in the thermograms of MSN-CUR and MSN-GA-CUR. These observations implied that the CUR was successfully incorporated into the mesoporous channels of MSN-CUR and MSN-GA-CUR and that CUR was present in a non-crystalline form.

\section{In vitro drug release}

The in vitro release profiles of CUR from MSN-CUR and MSN-GA-CUR in 30\% ethanol solution were investigated. As shown in Figure 6, only 12.9\% CUR was released within $24 \mathrm{~h}$ from pure CUR, which revealed the poor solubility of the pure CUR crystals. However, almost 50\% CUR was cumulatively released from MSN-CUR and MSN-GA-CUR within the first $8 \mathrm{~h}$. These results indicated that CUR became more soluble after incorporation into MSN or MSN-GA.

Referring to the results of the above DSC analysis, the significant difference in the release profiles between CUR and MSN-CUR-GA may be attributed to the incorporation of non-crystalline CUR into the mesopores of MSN or MSN-GA. Meanwhile, the contact area between the drug and dissolution medium increased after CUR was incorporated into the MSN or MSN-GA. These results accelerated the release of CUR from both MSN-CUR and MSN-GA-CUR.

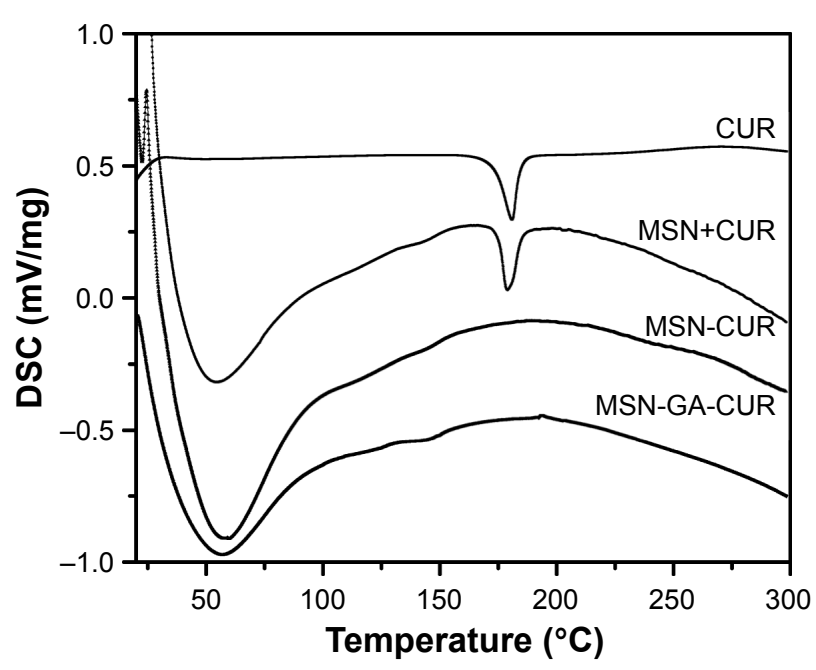

Figure 5 DSC curves of CUR, physical mixture of MSN and CUR (MSN+CUR), MSN-CUR and MSN-GA-CUR.

Abbreviations: CUR, curcumin; DSC, differential scanning calorimetry; GA, glycyrrhetinic acid; MSN, mesoporous silica nanoparticle.

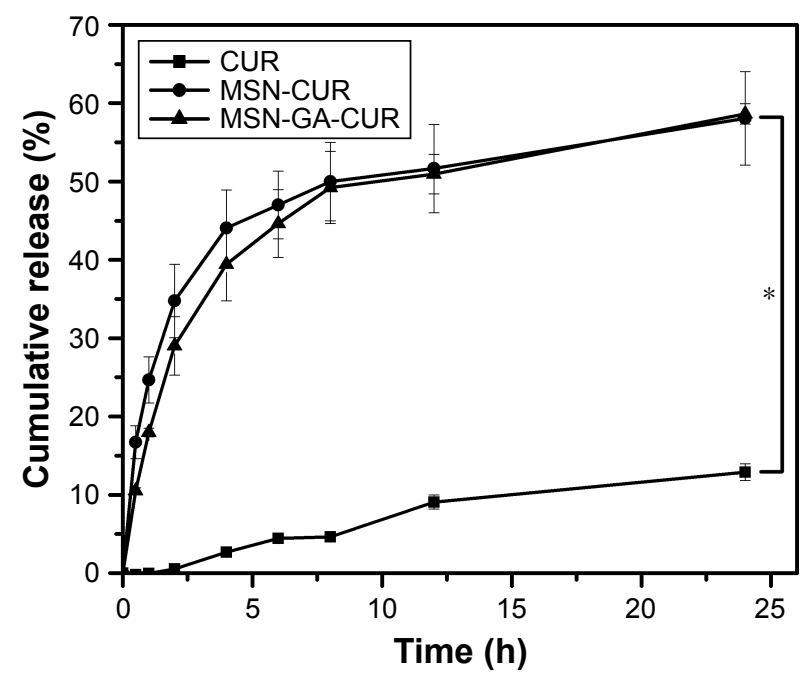

Figure 6 In vitro release of CUR from free CUR, MSN-CUR, and MSN-GA-CUR in $30 \%$ ethanol solution $(n=3)$.

Note: $* P<0.01$.

Abbreviations: CUR, curcumin; GA, glycyrrhetinic acid; MSN, mesoporous silica nanoparticle.

Moreover, the release of MSN-CUR and that of MSN-GACUR were not significantly different.

\section{Determination of nanoparticle cytotoxicity}

The cytotoxicity of MSN-GA against HepG2 cells was determined to investigate the cell targeting property of MSN-GA. As shown in Figure 7, all CUR formulations displayed dosedependent toxicity against HepG2 cells. Free CUR had obvious cytotoxicity against HepG2 cells. MSN-CUR showed relatively higher cytotoxicity against HepG2 cells, which may be attributed to the internalization of nanoparticles into

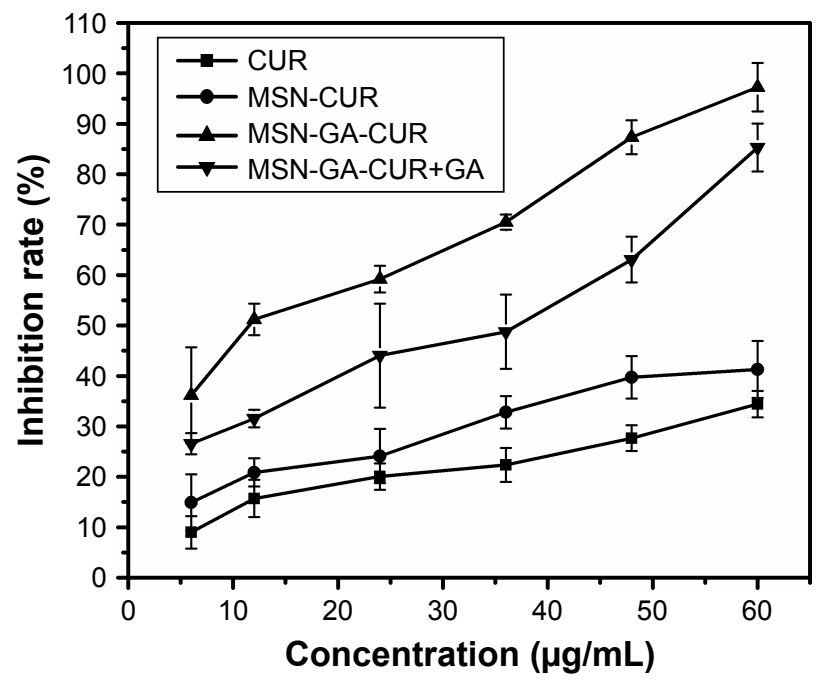

Figure 7 Cytotoxicity of various formulations against HepG2 cells after coincubation for $24 \mathrm{~h}$ at $37^{\circ} \mathrm{C}$.

Abbreviations: CUR, curcumin; GA, glycyrrhetinic acid; MSN, mesoporous silica nanoparticle. 


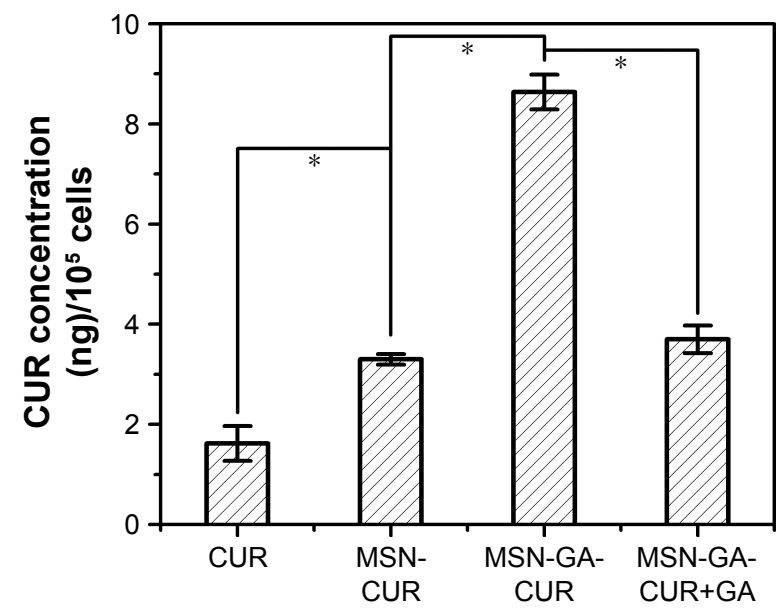

Figure 8 Cellular uptake of different CUR-loading formulations after $4 \mathrm{~h}$ of coincubation at $37^{\circ} \mathrm{C}$.

Notes: The CUR concentration was measured by HPLC. $* P<0.01$.

Abbreviations: CUR, curcumin; GA, glycyrrhetinic acid; HPLC, high-performance liquid chromatography; MSN, mesoporous silica nanoparticle.

cells via a non-specific mechanism. A similar trend but with a significantly higher degree was observed for MSN-GA-CUR. The mechanism involved in this phenomenon is most likely specific GA receptor-mediated endocytosis. Thus, a GA competitive binding assay was performed in parallel. Free $\mathrm{GA}(135.73 \mu \mathrm{mol} / \mathrm{mL})$ was added in advance before the addition of MSN-GA-CUR (MSN-GA-CUR+GA). The results of the GA competitive binding assay showed that the inhibitory effects of MSN-GA-CUR+GA on HepG2 cells were lower than that of MSN-GA-CUR. These effects could be caused by the competitive binding of free GA with GA receptors, thus decreasing the cellular uptake of MSN-GA-CUR. These results confirmed the specific GA receptor-mediated endocytosis mechanism of MSN-GA-CUR. This mechanism enhanced the cellular uptake of GA-modified nanoparticles, thus increasing the cytotoxicity of MSN-GA-CUR.

\section{In vitro cellular uptake}

The in vitro uptakes of different formulations by HepG2 cells were investigated with HPLC and CLSM methods. The results are displayed in Figures 8 and 9. After $4 \mathrm{~h}$ of coincubation, the cells internalized different degrees of all CUR formulations (Figure 8). Consistent with the cytotoxicity studies, MSN-GA-CUR showed a significantly higher cellular uptake than all other formulations. This effect resulted from
A

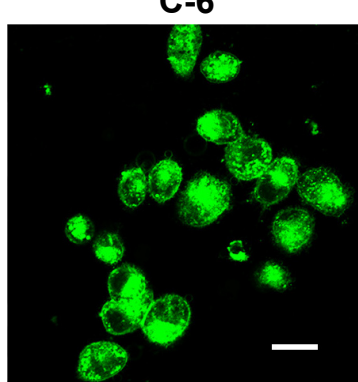

B

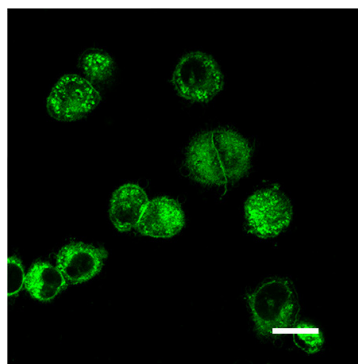

C

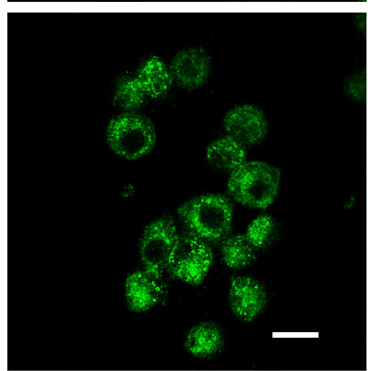

DAPI
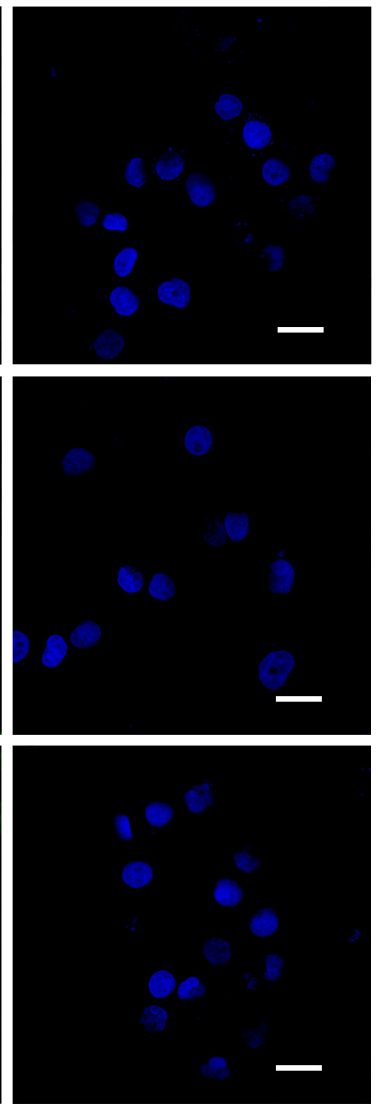

Merged
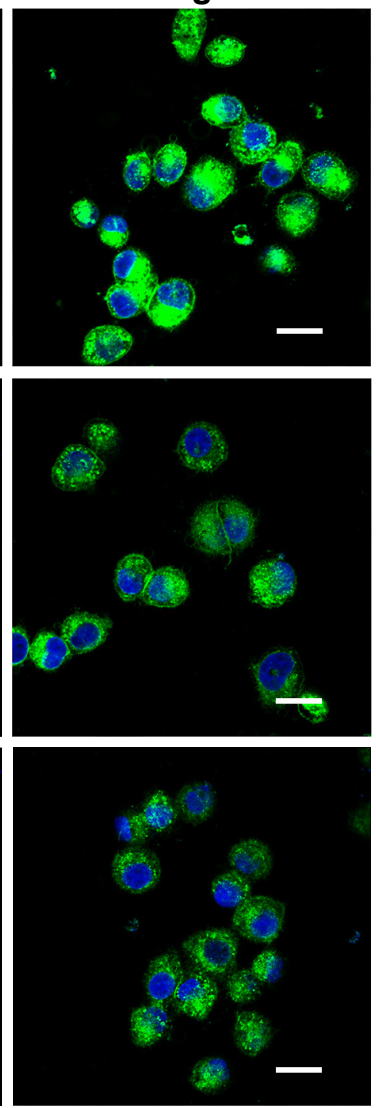

Figure $9 \mathrm{CLSM}$ images of HepG2 cells after 30 min of co-incubation with $100 \mu \mathrm{g} / \mathrm{mL}$ of C-6-MSN-GA (A), C-6-MSN (B), or C-6-MSN-GA with free GA (C) at 37 ${ }^{\circ} \mathrm{C}$. Scale bar: $20 \mu \mathrm{m}$. Magnification $\times 800$. C-6: C-6-MSN or C-6-MSN-GA (green), DAPI: cell nuclei (blue), merged: fluorescence overlaid image.

Abbreviations: CLSM, confocal laser scanning microscopy; DAPI, 6-diamidino-2-phenylindole; GA, glycyrrhetinic acid; MSN, mesoporous silica nanoparticle. 

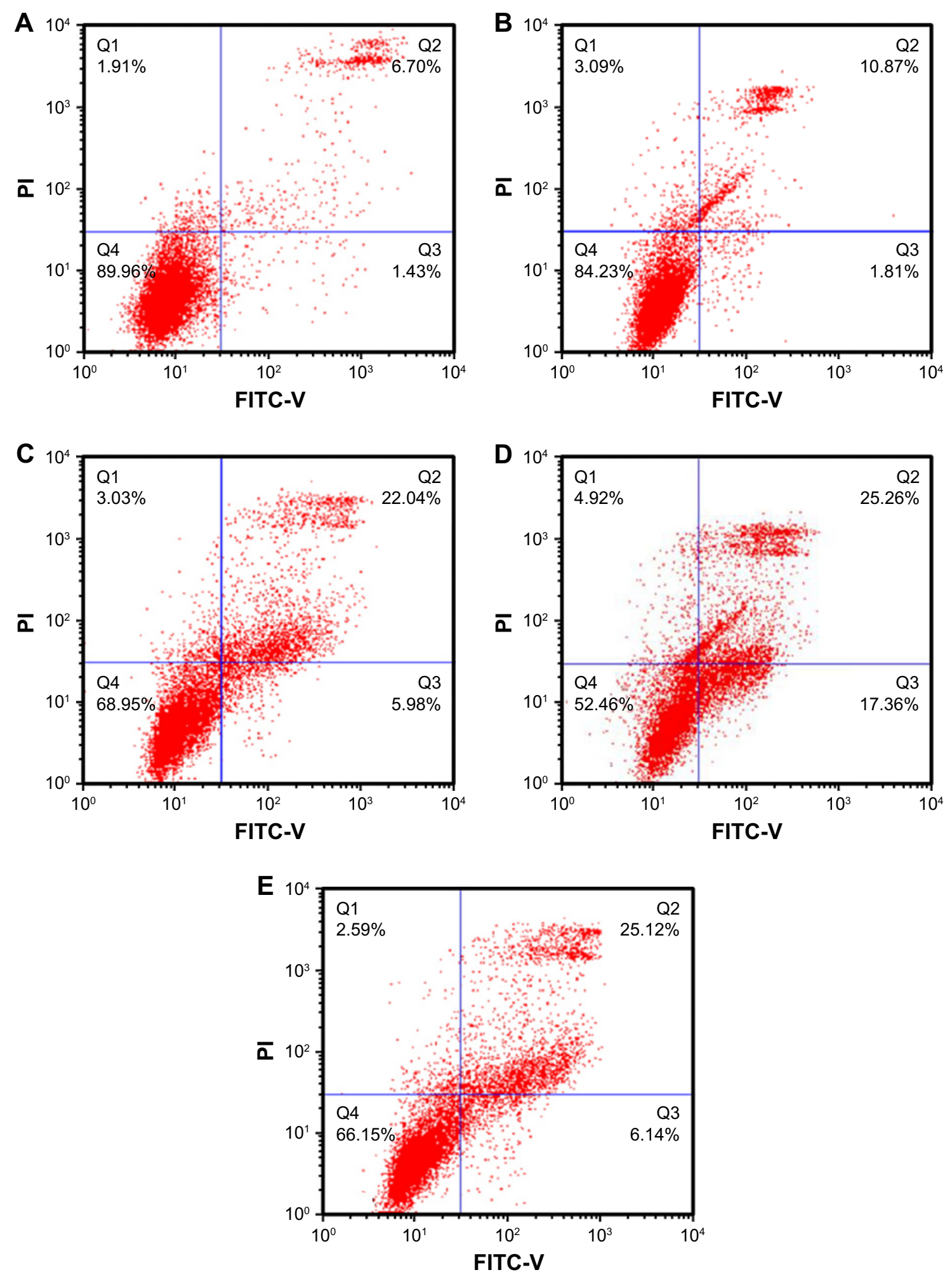

Figure 10 Effect of sterile PBS (A), free CUR (B), MSN-CUR (C), MSN-GA-CUR (D), and free GA with MSN-GA-CUR (E) on the induction of apoptosis in HepG2 cells. Abbreviations: CUR, curcumin; FITC-V, V-fluorescein isothiocyanate; GA, glycyrrhetinic acid; MSN, mesoporous silica nanoparticle; PI, propidium iodide.

the specific GA receptor-mediated endocytosis mechanism. Meanwhile, because free GA competitively bound to GA receptors, the cellular uptake of MSN-GA-CUR+GA relatively decreased compared with that of MSN-GA-CUR.
CLSM images (Figure 9) visually presented results that are similar to those of HPLC. Cells that were treated with different nanoparticles revealed different intensities of green fluorescence, which represented C-6-MSN or C-6-MSN-GA 
nanoparticles. An abundance of C-6-MSN-GA nanoparticles was internalized by HepG2 cells (Figure 9A) due to the specific GA receptor-mediated endocytosis mechanism as mentioned above. However, only a few C-6-MSN nanoparticles were internalized by HepG2 cells (Figure 9B), which may be attributed to non-specific uptake. Furthermore, consistent with the results of the HPLC method, the uptake of C-6-MSN-GA by HepG2 cells that were pretreated with free GA was significantly lower than that of C-6-MSN-GA alone. This result demonstrated that free GA could reduce the cellular uptake of C-6-MSN-GA by blocking the binding between C-6-MSN-GA and GA receptor.

These results further confirmed the specific GA receptormediated endocytosis mechanism and the cell targeting property of the GA-functionalized formulation.

\section{Apoptosis and cell cycle measurement}

To study the mechanism of CUR-induced cytotoxicity on HepG2 cells, FCM with FITC-V/PI double staining was employed to investigate cell apoptosis. Apoptosis rate was calculated as the sum of early (Q2) and late apoptosis (Q3). As shown in Figure 10, 8.1\% apoptotic cells were found in the control group, whereas $12.68 \%, 28.02 \%$, and $42.62 \%$ apoptotic cells were found in free CUR, MSN-CUR, and MSN-GA-CUR groups, respectively. The significantly increased apoptosis rate in MSN-GA-CUR treated cells again confirmed that GA-functionalized MSN-GA-CUR highly induced apoptosis in HepG2 cells.

The GA competitive binding assay was also performed. HepG2 cells that were pretreated with free GA exhibited an apoptosis rate of $31.26 \%$, which was lower than that of MSN-GA-CUR alone. These results are in agreement with the results for cellular uptake and cytotoxicity analyses. All observations demonstrated that the GA-functionalized MSN-GA-CUR highly induced apoptosis in HepG2 cells via a specific GA receptor-mediated endocytosis mechanism.

\section{Conclusions}

In summary, we exploited MSN-GA to carry CUR for active tumor targeting. MSN-GA not only exhibited a satisfactory loading capacity but also increased drug uptake by GA receptor-positive cells. The successful synthesis of MSN-GA was verified by FTIR, TEM, and zeta potential measurement. The results of cellular experiments demonstrated that MSNGA-CUR significantly enhanced cytotoxicity and cellular uptake toward GA-positive tumor cells via a specific GA receptor-mediated endocytosis mechanism. Therefore, the results of this study provide a promising nanoplatform for the targeting of hepatocellular carcinoma.

\section{Acknowledgments}

This project was supported by the Natural Science Foundation Project of CQ CSTC (No cstc2016jcyjA0068).

\section{Disclosure}

The authors report no conflicts of interest in this work.

\section{References}

1. Ma L, Ji L, Yu Y, Wang J. Novel molecular targets for diagnosis and treatment of hepatocellular carcinoma. Discov Med. 2015;19(102): $7-14$.

2. Wang YG, Huang PP, Zhang R, Ma BY, Zhou XM, Sun YF. Targeting adeno-associated virus and adenoviral gene therapy for hepatocellular carcinoma. World Journal of Gastroenterology. 2016;22(1):326-337.

3. Qi F, Li A, Inagaki Y, et al. Chinese herbal medicines as adjuvant treatment during chemo- or radio-therapy for cancer. Biosci Trends. 2010; 4(6):297-307.

4. Qian J, Feng GS, Vogl T. Combined interventional therapies of hepatocellular carcinoma. World J Gastroenterol. 2003;9(9):1885-1891.

5. Brannon-Peppas L, Blanchette JO. Nanoparticle and targeted systems for cancer therapy. Adv Drug Deliv Rev. 2004;56(11):1649-1659.

6. Bruckman MA, Randolph LN, Gulati NM, Stewart PL, Steinmetz NF. Silica-coated Gd(DOTA)-loaded protein nanoparticles enable magnetic resonance imaging of macrophages. J Mater Chem B Mater Biol Med. 2015;3(38):7503-7510.

7. Jhaveri A, Deshpande P, Torchilin V. Stimuli-sensitive nanopreparations for combination cancer therapy. J Control Release. 2014;190: 352-370.

8. Sun S, Liang N, Yamamoto H, Kawashima Y, Cui F, Yan P. pH-sensitive poly(lactide-co-glycolide) nanoparticle composite microcapsules for oral delivery of insulin. Int J Nanomedicine. 2015;10:3489-3498.

9. Liu D, Auguste DT. Cancer targeted therapeutics: From molecules to drug delivery vehicles. J Control Release. 2015;219:632-643.

10. Wang Y, Zhao Q, Han N, et al. Mesoporous silica nanoparticles in drug delivery and biomedical applications. Nanomedicine. 2015; 11(2):313-327.

11. Li X, Wu M, Pan L, Shi J. Tumor vascular-targeted co-delivery of anti-angiogenesis and chemotherapeutic agents by mesoporous silica nanoparticle-based drug delivery system for synergetic therapy of tumor. Int J Nanomedicine. 2015;11:93-105.

12. Yang Y, Yu C. Advances in silica based nanoparticles for targeted cancer therapy. Nanomedicine. 2016;12(2):317-332.

13. Lee H, Sung D, Kim J, et al. Silica nanoparticle-based dual imaging colloidal hybrids: cancer cell imaging and biodistribution. Int $J$ Nanomedicine. 2015;10 Spec Iss:215-225.

14. Melvin A, Vijay R, Chaudhari VR, et al. A facile methodology for the design of functionalized hollow silica spheres. J Colloid Interface Sci. 2010;346(1):265-269.

15. Wang Y, Du H, Zhai G. Recent advances in active hepatic targeting drug delivery system. Curr DrugTargets. 2014;15(6):573-599.

16. Negishi M, Irie A, Nagata N, Ichikawa A. Specific binding of glycyrrhetinic acid to the rat liver membrane. Biochim Biophys Acta. 1991; 1066(1):77-82.

17. He ZY, Zheng X, Wu XH, et al. Development of glycyrrhetinic acidmodified stealth cationic liposomes for gene delivery. Int J Pharm. 2010 397(1-2):147-154.

18. Cheng M, Chen H, Wang Y, et al. Optimized synthesis of glycyrrhetinic acid-modified chitosan 5-fluorouracil nanoparticles and their characteristics. Int J Nanomedicine. 2014;9:695-710.

19. Cutrignelli A, Lopedota A, Denora N, et al. A new complex of curcumin with sulfobutylether-beta-cyclodextrin: characterization studies and in vitro evaluation of cytotoxic and antioxidant activity on HepG-2 cells. J Pharm Sci. 2014;103(12):3932-3940. 
20. Xiao J, Chu Y, Li X, et al. Synthesis and biological analysis of a new curcumin analogue for enhanced anti-tumor activity in HepG 2 cells. Oncol Rep. 2010;23(5):1435-1441.

21. Xiao B, Si X, Han MK, Viennois E, Zhang M, Merlin D. Co-delivery of camptothecin and curcumin by cationic polymeric nanoparticles for synergistic colon cancer combination chemotherapy. J Mater Chem B Mater Biol Med. 2015;3(39):7724.

22. Margulis K, Srinivasan S, Ware MJ, Summers HD, Godin B, Magdassi S. Active curcumin nanoparticles formed from a volatile microemulsion template. J Mater Chem B Mater Biol Med. 2014;2:3745-3752.

23. Anand P, Kunnumakkara AB, Newman RA, Aggarwal BB. Bioavailability of curcumin: problems and promises. Mole Pharm. 2007;4(6): 807-818.

24. Cai Q, Lin WY, Xiao FS, Pang WQ, Chen XH, Zou BS. The preparation of highly ordered MCM-41 with extremely low surfactant concentration. Microporous Mesoporous Mater. 1999;32(1-2):1-15.
25. Jambhrunkar S, Qu Z, Popat A, et al. Effect of surface functionality of silica nanoparticles on cellular uptake and cytotoxicity. Mol Pharm. 2014;11(10):3642-3655.

26. Peng J, Sun Y, Zhao L, et al. Polyphosphoric acid capping radioactive/ upconverting NaLuF4:Yb,Tm,153Sm nanoparticles for blood pool imaging in vivo. Biomaterials. 2013;34(37):9535-9544.

27. Chen Z, Li Z, Lin Y, Yin M, Ren J, Qu X. Biomineralization inspired surface engineering of nanocarriers for $\mathrm{pH}$-responsive, targeted drug delivery. Biomaterials. 2013;34(4):1364-1371.

28. Slowing II, Vivero-Escoto JL, Wu CW, Lin VS. Mesoporous silica nanoparticles as controlled release drug delivery and gene transfection carriers. Adv Drug Deliv Rev. 2008;60(11):1278-1288.
International Journal of Nanomedicine

\section{Publish your work in this journal}

The International Journal of Nanomedicine is an international, peerreviewed journal focusing on the application of nanotechnology in diagnostics, therapeutics, and drug delivery systems throughout the biomedical field. This journal is indexed on PubMed Central, MedLine, CAS, SciSearch ${ }^{\circledR}$, Current Contents ${ }^{\circledR} /$ Clinical Medicine,

\section{Dovepress}

Journal Citation Reports/Science Edition, EMBase, Scopus and the Elsevier Bibliographic databases. The manuscript management system is completely online and includes a very quick and fair peer-review system, which is all easy to use. Visit http://www.dovepress.com/ testimonials.php to read real quotes from published authors. 